\title{
A Compact Wideband Dielectric Loaded H- plane Sectoral Ridged SIW Horn Antenna
}

\author{
Mahdi Reshadatmand1, Hamid Reza Hassani1, and Sajad Mohammad-Ali-Nezhad² \\ ${ }^{1}$ Electrical \& Electronics Engineering Department, Shahed University, Tehran, I. R. IRAN \\ ${ }^{2}$ Electrical \& Electronics Engineering Department, University of Qom, Qom, I.R. IRAN \\ Corresponding author: Hamid Reza Hassani (e-mail: hassani@shahed.ac.ir).
}

\begin{abstract}
In this paper a compact and wideband ridged substrate integrated waveguide (RSIW) horn antenna is proposed. Unlike the previous works, the ridge structure in the proposed antenna is tapered only along the width allowing the antenna to be designed on just two-layer dielectric substrate. The tapered ridge is created by simple, different diameter size vias just in one layer. The antenna designed has dimension of $14 \times 33 \times 3 \mathrm{~mm} 3\left(1.4 \lambda_{0} \times 3.3 \lambda_{0} \times 0.3 \lambda_{0}\right.$, where $\lambda_{0}$ is the free space wavelength at $\left.30 \mathrm{GHz}\right)$ and a has a bandwidth of $41.32 \%$ covering $24-36.5 \mathrm{GHz}$ with the reflection coefficient, S11, under $-10 \mathrm{~dB}$. The antenna is simulated through two software packages CST and HFSS and results are compared.
\end{abstract}

INDEX TERMS: Dielectric Load, Ridge, SIW Horn, Wideband.

\section{INTRODUCTION}

$\mathbf{O}$ ne of the simplest and widely used directive microwave antennas is the horn. Horn antennas in their usual form are bulky, expensive and take time to fabricate, especially at higher frequencies [1-4].

In the past few years, H-plane substrate integrated waveguide (SIW) horn antenna structure has been widely used [5-7]. Due to the use of printed circuit technology, SIW antennas are light in weight, compact in size, low profile, easy to integrate with other elements and are easy to fabricate.

Despite the above advantages, one of the limitations of the SIW horn antennas is their bandwidth. Dielectric loaded SIW H-plane horn antenna was introduced in [8], [9] to improve the bandwidth. In [8] an SIW horn antenna with an arc shaped lens of same permittivity as the rest of the structure placed in front of it, results in a better impedance matching providing $11 \%$ of bandwidth. [9] places a rectangular dielectric lens in the front.

To improve mismatch between the SIW horn antenna aperture and the free space, a printed transition is reported in [10], [11] providing $16.7 \%$ bandwidth.

In [12] a SIW H-plane horn antenna with 10 dielectric layers forming a tapered ridge in height is introduced giving $76 \%$ bandwidth (18-40 GHz) for VSWR below 2.5. The fabrication of antenna is quite difficult and expensive.

A complicated H-plane ridged SIW horn antenna is reported in [13] providing $92 \%$ of bandwidth with VSWR below 2.5. The structure uses 4 sections in front of each other: a three step tapered ridge, an arc-shaped tapered copper surface, and a cone shaped dielectric extended slab and an extended ground plane in front of the horn antenna.
Rather than using ridge to provide a higher bandwidth, [14] uses an extended air-via perforated (different hole diameters) dielectric section in front of the SIW horn antenna. The antenna gives $40 \%$ bandwidth $(16-24 \mathrm{GHz})$. It needs to be said that a rectangular waveguide is used in the feed section which makes the overall design complicated.

An air-via perforated SIW horn antenna is also reported in [16] with partially detached perforated broad walls in the flare part, providing $40 \%$ bandwidth $(17.7-26.7 \mathrm{GHz})$.

In [17], a wideband surface-mountable SIW horn antenna is reported in which the upper broad wall of conventional structure is removed and air-via is used. This antenna has $35.7 \%$ impedance bandwidth (19.1-27.4 GHz).

As can be seen from the above, the previous authors have either used a large number of dielectric layers, tapering of the ridge in height or in height as well as in width together or have placed a long perforated dielectric slab in front of the horn antenna in order to provide a large bandwidth.

In this paper, we present a compact wideband dielectric loaded H-plane sectoral ridged SIW horn antenna. The structure is based on two-layer dielectric substrate and uses a width tapered ridge horn antenna. The horn antenna has a simple dielectric loaded slab in the front section. The structure is very simple and can easily be fabricated. The proposed antenna structure is simulated through two software packages CST and HFSS. Results are provided and discussed.

\section{Antenna design}

The antenna structure proposed uses two dielectric substrates, the first and the second substrate with same permittivity. 
For the first substrate, the bottom of the substrate is grounded while on the top of the substrate exists a narrow conducting strip with varying width in which the tapered ridge vias are introduced from the top to the bottom of the substrate, Fig. 1a. The final feed structure will be attached to this tapered ridge.

A second substrate is then considered. On the top of this second substrate conducting layer is placed while on its bottom layer only dielectric is seen. This second substrate is then placed on the first substrate. From the top of the second substrate to the bottom of the first substrate, left and right side vias are introduced in order to create the waveguide side walls, Fig. 1b.

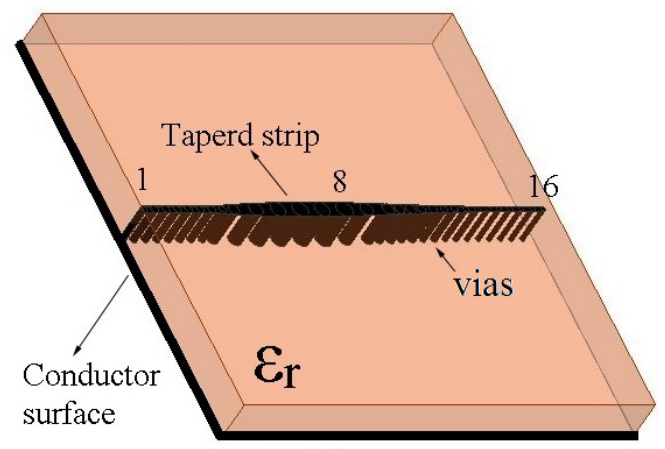

(a)

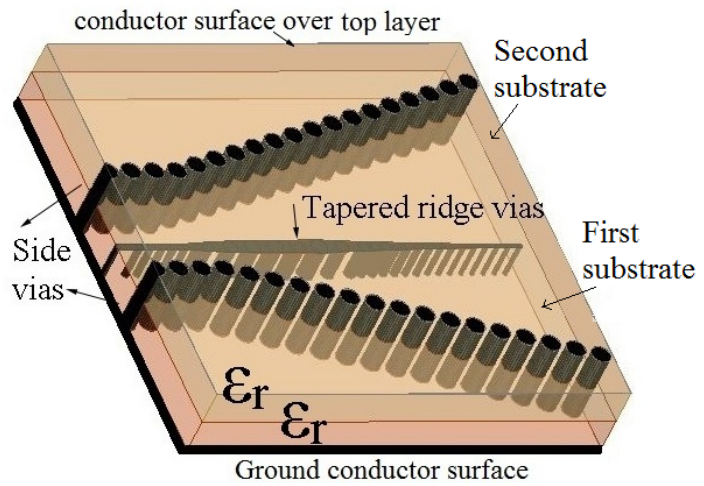

(b)

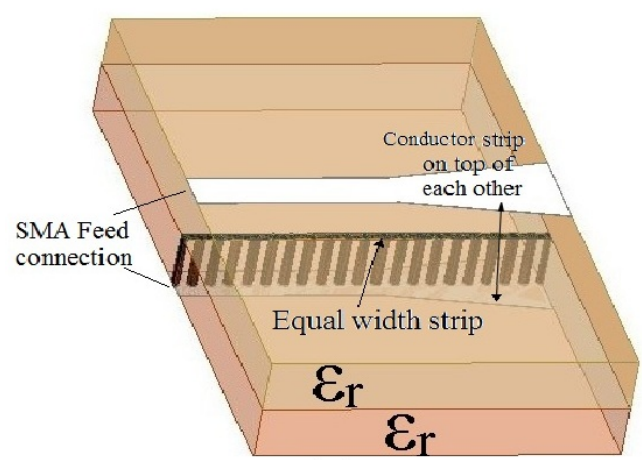

(c)

FIGURE 1. (a) The First substrate showing the tapered ridge (tapered strip on top of vias), (b) the second substrate on top of the first substrate with side vias from top to bottom and (c) feed section.

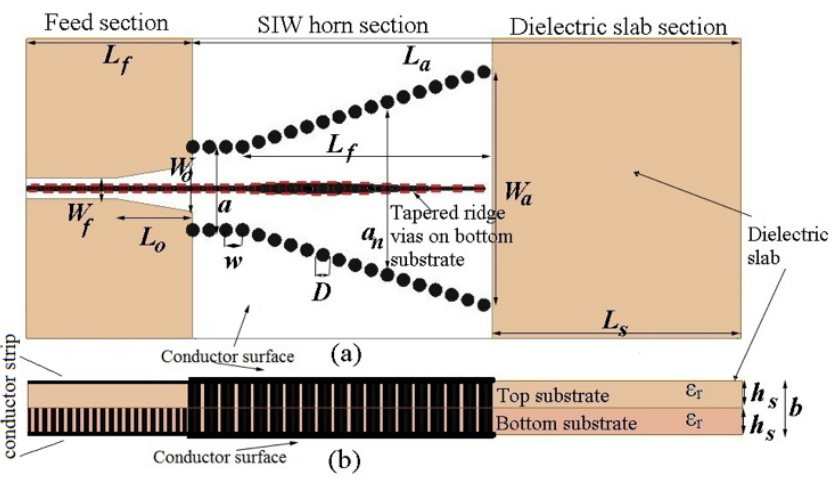

FIGURE 2. The Final structure of the proposed antenna. (a) top, (b) front and (c) side view of the proposed antenna. The vias are shown by black circles.

To improve the impedance bandwidth of the overall structure one can extend both the first and second substrates in the cross section and create the dielectric slab section, Fig. 2.

Fig. 2 shows the overall configuration of the proposed wideband ridged SIW horn antenna. The overall length of the designed horn is $L_{a}=33 \mathrm{~mm}$ and the width of the aperture is $W_{a}=14 \mathrm{~mm}$.

In the following subsections the design of each part of the proposed antenna is provided.

\section{A. SIW HORN STRUCTURE}

The overall antenna structure uses two dielectric substrates, the first and the second substrates, each of which has thickness $h_{s}=1.5 \mathrm{~mm}\left(h_{s}=b / 2\right)$ and dielectric constant of $\varepsilon r=4.8$.

Similar to an ordinary waveguide, the SIW horn section of the antenna, as shown in Fig. 2, uses top and bottom conductor surfaces on the dielectrics. Two linear equally spaced metal vias from top to bottom surfaces are used that act as electric wall making the excited mode inside the SIW to be approximately $\mathrm{TE}_{10}$ mode. One can define an equivalent waveguide for this SIW. As such, consider a rectangular wave-guide with cross section parameters $a$, and $b^{\prime}$ attached to a horn antenna. To have a single mode excitation of this horn antenna the dimensions $a$ ' and $b^{\text {' }}$ ( $a$ ': the width and $b^{\prime}$ : the height) should be chosen according to the following: [9]

$$
2 a^{\prime}>\lambda \sqrt{\mathcal{E}_{r}}>\max \left(a^{\prime}, b^{\prime}\right)
$$

The SIW horn section of the antenna has top and bottom metal surface and vias along the edges of the horn. This means that the wave within the horn section only sees the dielectric within the horn section (and no air).

The equivalent width at the input of the SIW horn section $a$ has been derived in [18] related to that of waveguide $a$ ' as:

$$
a=\frac{2 a^{\prime}}{\pi} \cot ^{-1}\left(\frac{\pi w}{4 a^{\prime}} \ln \frac{w}{4 R}\right)
$$


Where $w$ is the spacing between two adjacent vias and $R$ is radius of the via. Based on these, we have chosen $R=0.8$ $\mathrm{mm}(R=D / 2)$ and $w=1 \mathrm{~mm}$.

From (2), the width $a$ of the SIW becomes $5 \mathrm{~mm}$. The height of the SIW, $b$, is set equal to the height of the equivalent waveguide $b^{\prime}$. Through simulation, for a given waveguide aperture, it is found that to have maximum gain and have a good $\mathrm{S}_{11}(<-10 \mathrm{~dB})$ for the proposed antenna the dielectric substrates used for the SIW horn section can be extended by $L_{s}=15 \mathrm{~mm}$.

\section{B. RIDGE STRUCTURE WITHIN SIW HORN STRUCTURE}

To have a good impedance matching, the last step in the design of the antenna is the tapering of the ridge within the SIW horn structure, [19], [20].

Based on the above design parameters, through simulation it can be shown that the waveguide section at the entrance of the SIW horn structure (without the inner ridge) has a characteristic impedance $Z_{o}=81.87 \Omega$ while the equivalent characteristic impedance seen at the aperture end of the SIW horn structure (before the dielectric slab) $Z_{L}$ is $43.6 \Omega$. In this paper to provide good impedance transition between the start and end of the SIW horn section the ridge is exponentially tapered with respect to the $\mathrm{x}$ coordinate, Fig. 1a or $1 \mathrm{~b}$.

The exponential tapering impedance of the ridged SIW is chosen as:

$$
q=Z_{0} e^{K y}\left(0 \leq y \leq L_{f}\right)
$$

Where $L_{f}$ is the axial length of the flared horn. The coefficient $\mathrm{K}$ is calculated as:

$$
K=\frac{1}{L_{f}} \ln \left(\frac{Z_{L}}{Z_{0}}\right)
$$

Since there are 16 vias within the flared section of the horn, the tapering section of the ridge, $L_{f}$, is also divided into 16 subsections. The characteristic impedance of the tapered RSIW horn, $Z_{n}(y)$ for each subsection, as calculated from (3) and (4) are shown in Table 1. Also shown in Table 1, is the practical size of the vias for implementation. Simulation results shows that by rounding the diameter of the simulated vias to the nearest values not much change in impedances is seen.

To obtain the required ridge diameters for the $Z_{n}(y)$ for each of the SIW horn subsections (vias $n=1, . ., 16$ ) in Table 1 , we start from the first via in the flared section of the SIW horn section (with a specific width a1) and use an equivalent ridged SIW, a SIW that also includes a uniform ridge (equal diameters) whose diameter is varied in the simulation until the required $Z_{n=1}(y)$ is obtained for that subsection. This process is repeated by considering the next subsection, $\mathrm{n}$ on its own with width an. In this way the diameters for all the ridge subsections for the required $Z_{n}(y)$ are obtained. In this way the SIW horn section including the tapered ridge can be designed, called RSIW. In order to get a smoother impedance matching a tapered strip is also placed on the top of the first substrate with width similar to the diameters of the vias, Fig. 1a or 1b.The height of the ridge is kept constant at $h_{s}=1.5$ $\mathrm{mm}$ throughout the above design procedure.

TABLE I. Units for Magnetic Properties

\begin{tabular}{lccc}
\hline \hline $\begin{array}{c}\text { SIW horn } \\
\text { subsection } \\
\text { number }\end{array}$ & $\begin{array}{c}Z_{n}(y) \\
(\Omega)\end{array}$ & $\begin{array}{c}\text { Diameter of SIW } \\
\text { tapered ridges found } \\
\text { through simulation } \\
(\mathrm{mm})\end{array}$ & $\begin{array}{c}\text { Diameter of the } \\
\text { actual holes to } \\
\text { create the vias } \\
(\mathrm{mm})\end{array}$ \\
\hline 1 & 81.87 & 0.299 & 0.3 \\
2 & 78.51 & 0.459 & 0.5 \\
3 & 75.28 & 0.573 & 0.6 \\
4 & 72.18 & 0.633 & 0.7 \\
5 & 69.21 & 0.656 & 0.7 \\
6 & 66.36 & 0.643 & 0.7 \\
7 & 63.63 & 0.683 & 0.6 \\
8 & 61.02 & 0.541 & 0.6 \\
9 & 58.51 & 0.483 & 0.5 \\
10 & 56.1 & 0.483 & 0.5 \\
11 & 53.79 & 0.343 & 0.3 \\
12 & 51.58 & 0.287 & 0.3 \\
13 & 49.46 & 0.24 & 0.2 \\
14 & 47.42 & 0.206 & 0.2 \\
15 & 45.47 & 0.2 & 0.2 \\
\hline \hline
\end{tabular}

\section{FEED SECTION}

To feed the proposed SIW horn antenna, the structure of Fig. 2 has a feed section, shown in Fig. 1c with more details.

The feed section consists of two conductor strips placed on bottom of the first substrate and on top of the second substrate. Also, based on the dimension of the first via of the tapered ridge within the SIW horn section, a number of similar vias equally spaced are placed in the first substrate between the two conductor strips, Fig. 1c.

The inner pin of an SMA connector is attached to the top conductor strip and the ground of the SMA connector is connected to the lower conductor strip. The conductor strip width $W_{f}$, Fig. 2, is adjusted through simulation to give $50 \Omega$ similar to that of SMA. Then, the conductor strip width is increased linearly until the impedance seen at the beginning of the conductor strip shows impedance similar to that of the SIW horn section. The main reason that the vias in the feed section are used is so that when wave enters the SIW horn section does not abruptly see the tapered ridge vias. The design procedure for such a feed (without the equal width ridge vias) is given in [21].

The final parameters used for the feed section are: $W_{f}=$ $1.29 \mathrm{~mm}, W_{o}=2.8 \mathrm{~mm}, L_{o}=4.5 \mathrm{~mm}$ and $L_{f}=10 \mathrm{~mm}$.

\section{SIMULATION RESULTS}

In this section the results of simulation of the proposed RSIW antenna via two software packages CST 2017 (a time domain solver) and HFSS package version 15.0 (a frequency domain solver) are presented and results are compared and 
discussed. The computer system used for simulation is Core i7 with 8 MB RAM.

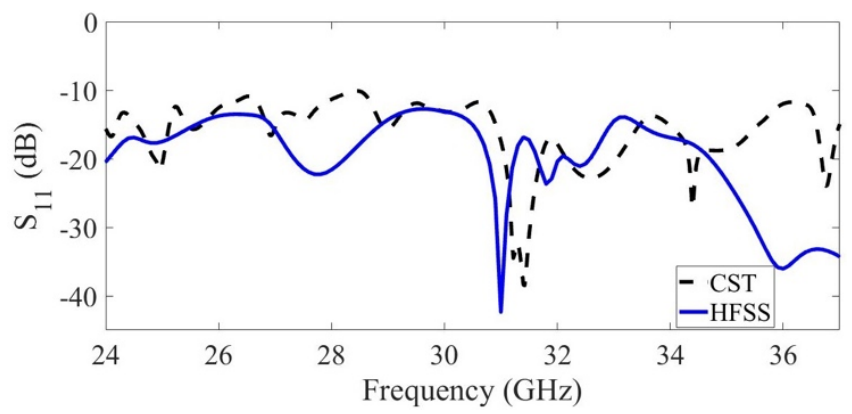

FIGURE 3. Simulated reflection coefficient of Linear and exponentially tapered horn opening

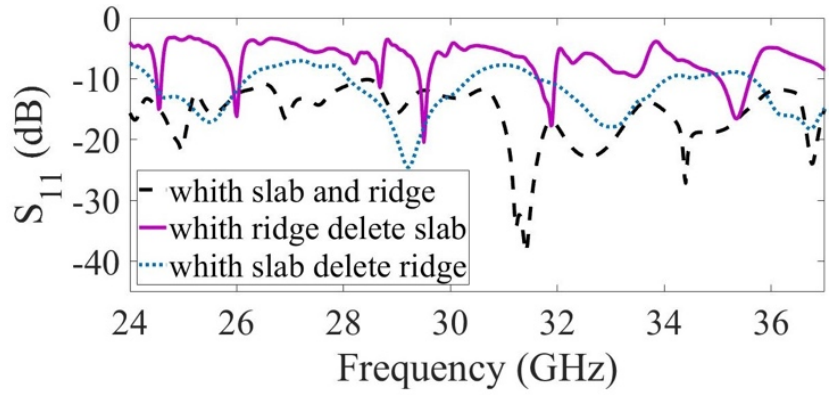

FIGURE 4. Simulated reflection coefficient of the antenna obtained through CST.

Throughout the analysis, the following specification for each package is used:

HFSS simulator uses Ansoft TAU mesh, and a distance of $\lambda / 4$ (based on lowest frequency of operation) between the $\mathrm{ABC}$ and the radiating structure is considered, and set for convergence at delta $\mathrm{S}=0.02$. The radiation box placed around the antenna structure is filled with air rather than vacuum for better accuracy. For excitation of the structure a wave port is considered. In the analyses the frequency sweeping is set to Discrete. Discrete mode compared to Fast sweeping mode results in higher accuracy but at the expense of higher computation time.

CST simulator uses Tetrahedron mesh without using the symmetry planes, automatically chooses boundary conditions based on the frequency of the analysis and set for a minimum of 329280 mesh cells. For excitation of the structure a wave port is considered.

Similar to [22] one can see the effect of the tapering of the horn opening on the S11 of the antenna. To see what effect the shape of the opening of the horn has on the reflection coefficient results, Fig. 3 shows the S11 for both a linear tapered and also for an exponentially tapered horn opening. Results shows that the linear taper provides a better $-10 \mathrm{~dB}$ impedance matching level, as such, throughout the paper the linear tapering is used, SIW horn section of Fig. 2.

Fig. 4 shows the reflection coefficient of the proposed structure with and without the dielectric slab or ridge as obtained through software CST. It is obvious that the impedance matching in the two cases that either dielectric slab or the ridge is not present is not appropriate. The structure in which both ridge and dielectric slab are present results in a good impedance matching throughout the bandwidth 24-37 GHz.

To confirm the accuracy of the results the reflection coefficient, S11, of the proposed RSIW horn antenna of Fig. 2 as obtained through CST and HFSS are shown in Fig. 5. It is observed that, the simulated bandwidth of $|\mathrm{S} 11|$ under -10 $\mathrm{dB}$ is $24-36.5 \mathrm{GHz}$ and also it is seen that there is good agreement between the simulation results of the two software packages.

Fig. 6 shows the far-field E-plane (y-z plane) and the Hplane ( $\mathrm{x}-\mathrm{y}$ plane) radiation patterns of the proposed RSIW antenna at four typical frequencies within the bandwidth, 25, 29, 33 and $36 \mathrm{GHz}$. It is observed that good agreement between the simulation results of the two software packages is obtained. It is obvious that the proposed antenna has stable radiation pattern.

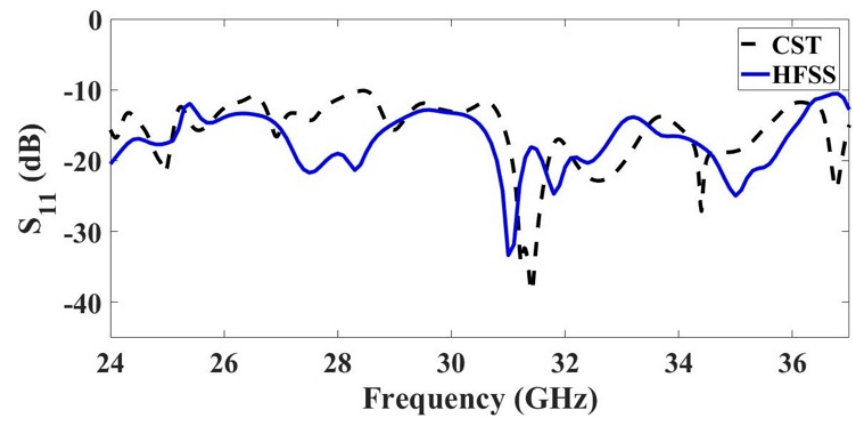

FIGURE 5. Simulated reflection coefficient of the proposed antenna as obtained through both CST and HFSS packages.

Although not shown, a study of the H-plane radiation patterns with ridge present or not gives the following observations. Side lobe level of the proposed antenna without the ridge is $-12.1 \mathrm{~dB}$ while with the ridge it becomes $-7.6 \mathrm{~dB}$. The Angular width of antenna without ridge is $28.77 \mathrm{o}$ and with ridge is 30.60 . Back lobe level is $-13.87 \mathrm{~dB}$ without ridge and $-13.2 \mathrm{~dB}$ with ridge. Again, although not shown, the cross polar of the proposed RSIW antenna is $-40 \mathrm{~dB}$.

The peak gain of the proposed antenna in the main beam direction with and without the ridge for various frequencies is shown in Fig. 7a has obtained through CST. It is seen that the gain is reduced when the ridge is present, and this can be due to the losses associated with the ridge. 


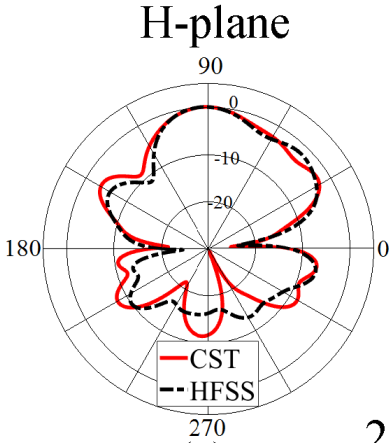

(a)

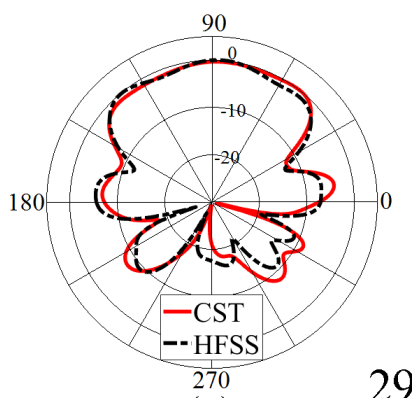

(c)

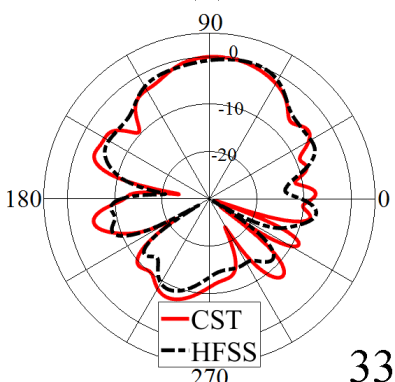

(e)

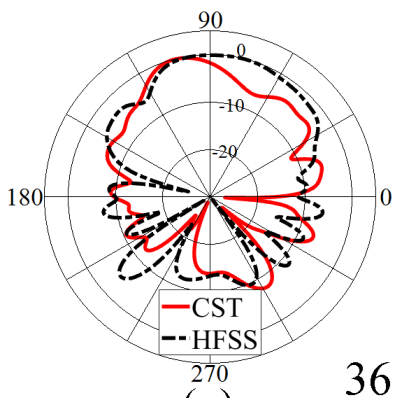

(g)

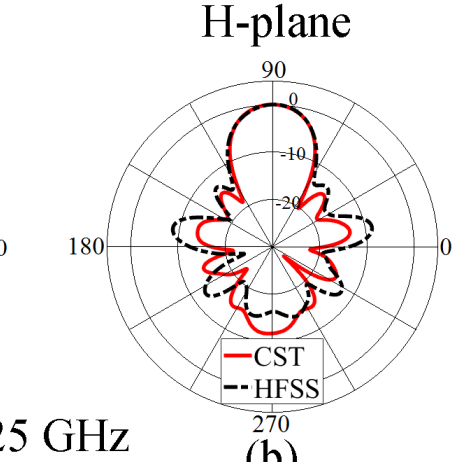

(b)

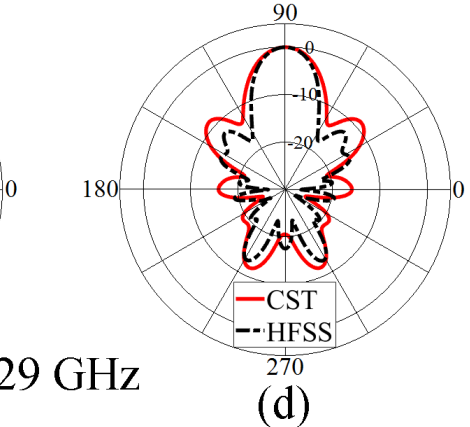

(d)

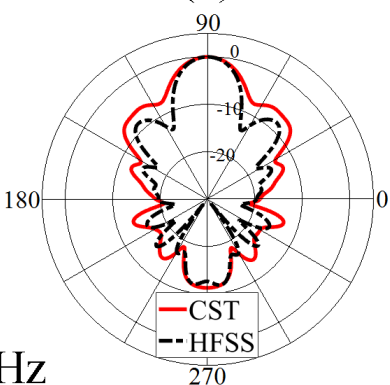

(f)

(h)

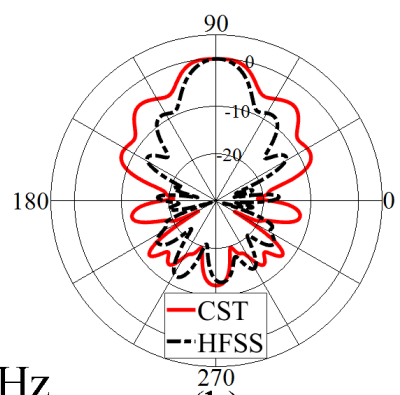

FIGURE 6. Normalized E- and H-plane radiation patterns of the proposed antenna at 25, 29, 33 and $36 \mathrm{GHz}$.

Table II gives a comparison between performance of various antenna structures reported in the literature and the present proposed structure. Our proposed antenna is comparable to that of [14]. It needs to mention that our structure is much simpler to construct than that of [14]. [14] uses a rectangular waveguide in the feed section as well as uses various diameter size vias in the dielectric slab section which make the structure complex.

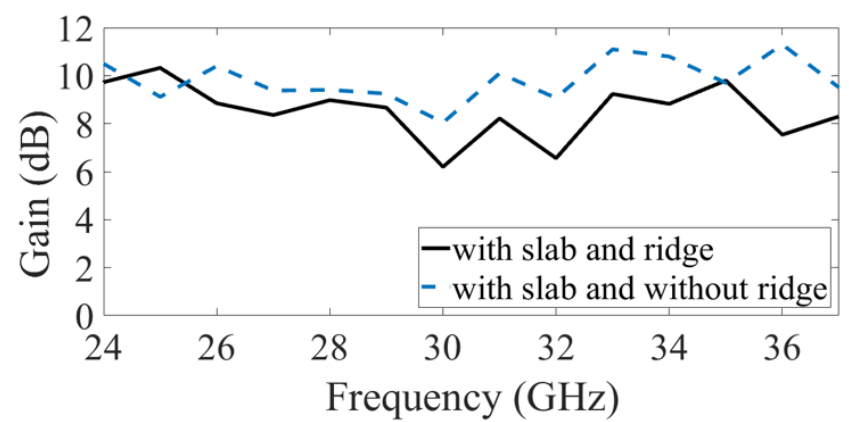

(a)

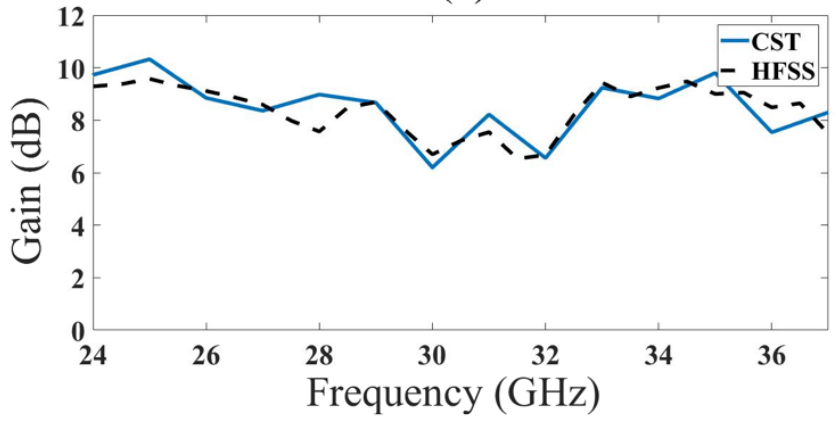

(b)

FIGURE 7. Simulated peak gain of the proposed antenna (a) CST simulation results of ridge being present or not, (b) comparison of CST and HFSS results of the proposed antenna with ridge present. .

TABLE II. Antenna Comparison

\begin{tabular}{ccccc}
\hline \hline Ref. & $\begin{array}{c}\text { Dimensions } \\
\mathrm{W} \times \mathrm{L} \times \mathrm{H}\left(\lambda_{0}\right)\end{array}$ & $\begin{array}{c}\text { Bandwidth } \\
\%\end{array}$ & $\begin{array}{c}\text { No. of } \\
\text { layers }\end{array}$ & $\left|\mathrm{S}_{11}\right|(\mathrm{dB})$ \\
\hline$[12]$ & $3.8 \times 6.7 \times 0.25$ & $\approx 76$ & 10 & $\mathrm{~S}_{11} \leq-7.36$ \\
{$[13]$} & $2.3 \times 5 \times 0.13$ & $\approx 92$ & 4 & $\mathrm{~S}_{11} \leq-7.36$ \\
{$[14]$} & $1.7 \times 4.1 \times 0.3$ & $\approx 40$ & 1 & $\mathrm{~S}_{11} \leq-10$ \\
{$[15]$} & $1.7 \times 3.9 \times 0.3$ & $\approx 31$ & 2 & $\mathrm{~S}_{11} \leq-10$ \\
$\begin{array}{c}\text { This } \\
\text { paper }\end{array}$ & $1.4 \times 3.3 \times 0.3$ & $\approx 41$ & 2 & $\mathrm{~S}_{11} \leq-10$ \\
\hline \hline
\end{tabular}

\section{CONCLUSION}

A compact and wideband dielectric loaded H-plane sectoral ridged substrate integrated waveguide (RSIW) horn antenna has been presented. To increase the bandwidth, the tapered ridge structure is tapered only along the width allowing the antenna to be designed on a two-layer dielectric substrate. A tapered ridge is made by simple different diameter size vias. The antenna has a bandwidth of $41.32 \%$ covering $24-36.5$ GHz. The proposed antenna has acceptable gain, back lobe level and cross polarization over the operating frequency. 


\section{REFERENCES}

[1] E. Rahimi and M. H. Neshati, "Development Of An enhanced Gain Substrate Integrated Waveguid H-Plane Horn Antenna Using Thin Substrate," International Journal of Engineering, vol. 29, no. 8, pp. 1062-1067, 2016.

[2] A. R. Mallahzadeh and A. A. Dastraj, "Double- ridged conical horn antenna for 2-18 GHz," Electromagnetics, vol. 28, pp. 450-461, 2008 .

[3] R. Manikandan, P. H. Rao, and P. K. Jawahar, "Gain Enhancement of Horn Antenna Using Meta Surface Lens," Advanced Electromagnetics, vol. 7, no. 4, pp. 27-33, Aug. 2018

[4] M. Carkaci and M. Secmen, "The Prototype of a Wideband Ku-Band Conical Corrugated Horn Antenna with 3-D Printing Technology", Advanced Electromagnetics, vol. 8, no. 2, pp. 39-47, Mar. 2019.

[5] A.R. Mallahzadeh and S. Mohammad-Ali-Nezhad,"Long Slot Ridged SIW Leaky Wave Antenna Design Using Transverse Equivalent Technique," IEEE Transaction on antenna and propagation, vol. 62, no. 11, pp.5445-5452, Nov. 2014

[6] A.R. Mallahzadeh and S. Mohammad-Ali-Nezhad,"A Low CrossPolarization Slotted Ridged SIW Array Antenna Design with Mutual Coupling Considerations," IEEE Transaction on antenna and propagation, vol. 63, no. 10, pp. 4324- 4333, Oct. 2015.

[7] Y. Cai, Y. Zhang, L. Yang, Y. Cao and Z. Qian, "Design of LowProfile Metamaterial-Loaded Substrate Integrated Waveguide Horn Antenna and Its Array Applications," IEEE Transactions on Antennas \& Propag., vol. 65, no. 7, pp. 3732-3737, Jul.

[8] W.-Q. Che, B. Fu, P. Yao, Y. L. Chow, and E. K. N. Yung, "A compact substrate integrated waveguide H-plane horn antenna with dielectric arc lens: Research articles," Int. J. RF Microw. Comput. Aided Eng., vol. 17, no. 5, pp. 473-479, Sep. 2007.

[9] H. Wang, D.-G. Fang, B. Zhang, and W.-Q. Che, "Dielectric loaded substrate integrated waveguide (SIW) H-plane horn antennas," IEEE Trans. Antennas Propag., vol. 58, no. 3, pp. 640-647, Mar. 2010.

[10] M. E. Morote, B. Fuchs, J.-F. Zurcher, and J. R. Mosig, "Novel thin and compact h-plane siw horn antenna," IEEE Transactions on Antennas \& Propag., vol. 61, no.6, pp. 2911-2920, Mar. 2013.

[11] M. E. Morote, B. Fuchs, J.-F. Zurcher, and J. R. Mosig. , "A printed transition for matching improvement of SIW horn antennas," IEEE Trans. Antennas Propag. , vol. 61, no. 4, pp. 1923-1930, Apr. 2013.
[12] A. R. Mallahzadeh and S. Esfandiarpour, "Wideband H-plane horn antenna based on ridge substrate integrated waveguide (RSIW)," IEEE Antennas \& Wireless Propag. Lett. vol. 11, pp. 85-88, Jan.2012.

[13] Y. Zhao, Z. X. Shen, and W. Wu, "Wideband and low-profile Hplane ridged SIW horn antenna mounted on a large conducting plane," IEEE Trans. Antennas Propag., vol. 62, no.11, pp. 58955900, Sep. 2014.

[14] Y. Cai, Z.-P. Qian, Y.-S. Zhang, J. Jin, and W.-Q. Cao, "Bandwidth enhancement of SIW horn antenna loaded with air-via perforated dielectric slab," IEEE Antennas Wireless Propag. Lett. vol. 13, pp. 571-574, Apr. 2014

[15] Cai, Y., Z. Qian, W. Cao, Y. Zhang, J. Jin, and L. Yang, "Compact Wideband SIW Horn Antenna Fed by Elevated-CPW Structure," IEEE Trans. Antennas Propag., vol. 63,no. 10, pp. 4551-4557, Jul. 2015.

[16] Y. Cai, Y.-S. Zhang, Z.-P. Qian, W.-Q. Cao, and L. Wang, "Design of compact air-vias Perforated SIW horn antenna with partially detached broad walls," IEEE Trans. Antennas Propag., vol. 64, no. 6 , pp. 2100-2107, Jun. 2016.

[17] Y. Cai, Z.-P. Qian, Y.-S. Zhang, L. Yan, Y. Cao, and Z. Qian, “A Low-Profile Wideband Surface-Mountable Substrate-Integrated Waveguide Horn Antenna," IEEE Antennas Wireless Propag. Lett. vol. 16, pp. 2730-2733, Oct. 2017.

[18] W. Che, K. Deng, D. Wang, and Y. L. Chow, "Analytical equivalence between substrate-integrated waveguide and rectangular waveguide," IET Microw. Antennas Propag., vol. 2, no. 1, pp. 35 41, Feb. 2008

[19] C. A. Balanis, Antenna Theory-Analysis and Design, 3rd ed NewYork, NY, USA: Wiley, 2005.

[20] D. M. Pozar, Microwave Engineering, second. New York: Wiley, 1998.

[21] Muhammad Imran Nawaz, Zhao Huiling, Muhammad Kashif, "Substrate Integrated Waveguide (SIW) to Microstrip Transition a X-Band",Pg 61-63, Proceedings of the 2014 International Conference on Circuits, Systems and Control.

[22] De Oliveira, A.M., Perotoni, M.B., Kofuji, S.T., et al: 'A palm tree antipodal Vivaldi antenna with exponential slot edge for improved radiation pattern', IEEE Antennas Wirel. Propag., 2015, 14, pp. 1334-1337. 